\title{
Notes and Comment
}

\section{Comparing strong and weak models by fitting them to computer-generated data}

\author{
CHARLES E. COLLYER \\ University of Rhode Island \\ Kingston, Rhode Island
}

In psychology, as in other sciences, theoretical models are sometimes constructed in order to give explicit expression to hypotheses about how things work. Choosing among alternative hypotheses may be approached from a variety of perspectives: one way is to fit appropriate models to some data and see which model fits best. It is quite unsurprising to learn that one theoretical model has been adopted in preference to other possible models on the grounds that its predictions conformed best to a set of empirical observations. We are quite accustomed to this reasoning; indeed, it is very reasonable to expect that the "true" model (if there is one, in a meaningful sense) would fit the data very well because of its truth. Superior fit, however, can come about in at least one other way, and that is through the sheer complexity of the model that the scientist entertains. This paper illustrates the inferential problem posed by this second means of achieving good fit, and demonstrates how appropriate control information can be gathered to put inferences from model-fitting on firmer ground.

\section{The Strength/Fit Tradeoff}

A "strong" theoretical model is a simple model; predictions about performance are generated on the basis of very few assumptions. A "weak" model is more complex; it embodies more assumptions about the processes underlying observable performance, and these additional assumptions are reflected in its predictions. The principle of parsimony in science dictates that strong models are preferable to weak models, other things being equal. That is, if the predictions of two models fit the available data equally well, the simpler model is preferred. However, strong and weak models rarely fit equally well; usually, weak models fit better. The strength and the fit of theoretical models thus trade off, sometimes forcing researchers to decide between a strong model that fits moderately well and a weak model that fits better.

One may opt for good fit above all else, and choose the model that conforms most closely to the data. The

\footnotetext{
I thank Joseph S. Rossi for valuable advice and comment during the preparation of this paper. An anonymous reviewer of an earlier draft provided very constructive criticism and helped to place the work in perspective. The thirty "stat-dogs" of Bush and Mosteller (1955), wherever they be now, were also sources of inspiration. Address correspondence to Charles E. Collyer, Department of Psychology, University of Rhode Island, Kingston, RI 02881.
}

main problem with this choice is termed "capitalization on error": when a model is fit to the set of data from which its parameters were estimated, the model is made to conform not only to the "true values" of the scores, but also to the particular error components of these scores. In general, because of the complex model's larger number of free parameters, a complex model capitalizes on error to a greater extent than a simple model. Each parameter estimated from the data brings the predictions of the model closer to the scores themselves. An extreme and well-known example is that, in curvilinear regression (see Pedhazur, 1983), a set of $k$ data points can be fit perfectly by a polynomial of order $k-1$ (the degrees of freedom in the data set). Now if you already had reason to believe that the data had been generated by a very simple process, you probably would not place much credence in a model so complex as to use up all or almost all available degrees of freedom. But what if instead you were hunting for a model to characterize that data? A policy of "good fit above all else" can lead to the error of adopting models that are both unnecessarily complex and theoretically empty.

Another strength/fit option is to choose the strongest model available. It is rare, however, for researchers to adopt and defend the literally strongest model, which is often the one-parameter null hypothesis (score = $\mu+$ error, where $\mu$, the parameter, is the population mean). Aside from the null model, a preference for strong models is more typically expressed when the researcher opts for reasonable parsimony and adopts the strongest model that fits well. The potential error that attends this bias is that a theoretically meaningful but more complex model may be neglected.

This paper reports two studies done as part of an investigation of the question, Is there a threshold for mental rotation? (Other experiments are reported by Rossi \& Collyer, in press.) Both of the threshold models of mental rotation considered here have more parameters than the nonthreshold model, and so may be expected to fit well whether they are "true" or not. However, we wanted to compare the models fairly, that is, in a way that partialed out the influence of model complexity. Hence, we were initially led to confront the general problem of comparing strong and weak models in order to address the mental rotation question.

\section{Mental Rotation}

In a mental rotation task, observers are shown a standard figure (typically a line drawing of a structure made of blocks) and a comparison figure; the two differ in angular orientation. To a close approximation, the average time required to recognize that the two figures are the same, rather than mirror images of each other, is a linear 
function of the angular disparity in the portrayed orientations of the two figures (Shepard \& Metzler, 1971). The linearity of the response-time function suggests that in comparing the shapes of two similar objects differing in orientation, an observer passes through a series of internal (mental) states bearing a one-to-one relation to physical states that the object would pass through if it were physically rotated from one orientation to the other (Cooper \& Shepard, 1978). The principal debate surrounding mental rotation research has been between those who accept the data as evidence for such a transformation of image-like representations and those who do not (e.g., Pylyshyn, 1979). The concerns of the present paper are independent of this debate, involving questions only about the detailed shape of the mental rotation responsetime function, not about mental representation per se. (For convenience, however, a vocabulary with image/rotation connotations is used.)

At the present time, the mental rotation task seems to be understood in terms of two mechanisms, one that rotates the internal representation into standard orientation and a second that then makes a comparison resulting in a "same" or "different" judgment. This conception of the process seems to require mental rotation prior to comparison for any nonzero angular disparity between the two figures. There is some lack of rigor here, but let us call this conception Model I. Assuming that mental rotation takes place at a constant rate, Model I predicts a strictly linear relationship between response time and angular disparity, as shown in the top panel of Figure 1. The parameters of Model I are i, the intercept, and s, the slope, of this function.

The implication in Model I that all nonzero angular disparities must be rotated is somewhat implausible. There may be a range of small angular disparities for which mental rotation prior to comparison is unnecessary. The upper limit of this range would then constitute a "threshold" for mental rotation. A threshold would imply that the comparison process is "'tolerant" to some extent. That is, the process would allow for immediate comparison not just for zero disparity, but also for the range of subthreshold angular disparities; the delay before comparison caused by mental rotation would occur only for suprathreshold disparities. In other areas of perception, such margins of tolerance and corresponding threshold measures are quite familiar. (However, it would be easy to miss a mental rotation threshold of $30^{\circ}$ or less, because most studies have not used small, nonzero disparities.) There are a number of ways to conceptualize the role of a threshold in the mental rotation task. Let us consider two threshold models which, although more complex than Model I, are still relatively simple and tractable.

Model II asserts that there is a threshold, and that rotation always results in the reduction of suprathreshold disparities to standard orientation prior to comparison. Thus, the subthreshold response time is equal to the intercept of the suprathreshold linear function, as shown in the middle panel of Figure 1. The parameters of Model II are $t$, the threshold for mental rotation, $i$, the intercept of the

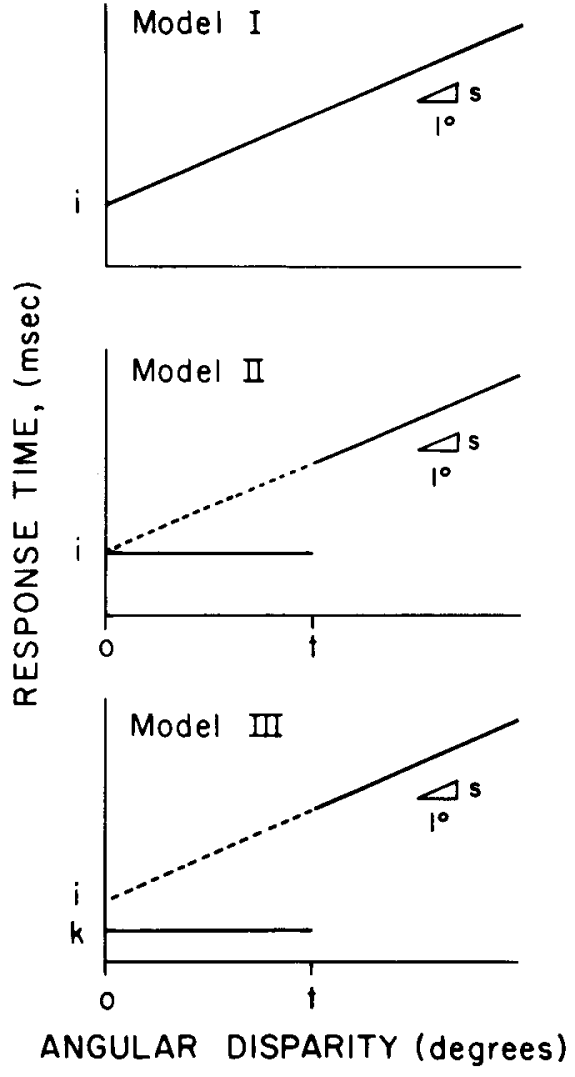

Figure 1. Forms of the mental rotation response-time function for three models.

suprathreshold linear function, and s, the slope of the suprathreshold linear function.

Model III allows for some additional processing complexities. If activation of the rotation process takes some time, or if suprathreshold disparities are rotated only enough to bring the comparison image within the subthreshold range, then the subthreshold response time may be either lower or higher than the intercept of the suprathreshold line. The response-time function of Model III is shown in the bottom panel of Figure 1. The parameters are $k$, the subthreshold response time, $t$, the threshold, $i$, the intercept of the suprathreshold line, and $\mathrm{s}$, the slope of the suprathreshold line.

\section{STUDY I: \\ Conventional Goodness of Fit}

Each of the three models was fit to mental rotation data collected by Rossi (1980) and reported by Rossi and Collyer (in press). To address the threshold question, Rossi had used trend analysis of group data (as opposed to modeling of individual subjects' performance) and heavily sampled a range of small angular disparities, from $0^{\circ}$ to $30^{\circ}$.

\section{Method}

Rossi's data consisted of sets of mean response times from 20 subjects. Only "correct, same" response times were included: that 
is, trials on which a subject gave the wrong response and trials on which the two stimulus figures differed in shape as well as in angular orientation were excluded. Each subject's set of 16 response times consisted of one for each of the following angles of comparison-figure rotation in the picture plane: $0^{\circ}, 3^{\circ}, 6^{\circ}, 9^{\circ}, 12^{\circ}$, $15^{\circ}, 18^{\circ}, 21^{\circ}, 24^{\circ}, 27^{\circ}, 30^{\circ}, 60^{\circ}, 90^{\circ}, 120^{\circ}, 150^{\circ}$, and $180^{\circ}$.

Models were fit to the data of each subject. Simple linear regression was used to estimate $i$ and $s$ for Model I. The proportion (P) of variance accounted for by Model I is given by the coefficient of determination, $\mathbf{r}^{2}$. Model II was fit by computing, for each candidate value of $t$ from $0^{\circ}$ to $30^{\circ}$ in $3^{\circ}$ increments, the linearregression trial values of $i$ and $s$ for all larger values of angular disparity. Each set of parameter values was used to predict the subject's 16 response times, and the residual sum of squares served as a measure of prediction error. The set of parameter values that maximized the proportion $(P)$ of total variance accounted for comprised the estimates of $\mathrm{i}, \mathrm{s}$, and $\mathrm{t}$ for this model. Model III was fit by computing, for each candidate value of $t$ from $0^{\circ}$ to $30^{\circ}$ in $3^{\circ}$ increments, the mean of response times up to and including the candidate threshold (giving a trial value of $k$ ) and the linearregression trial values of $i$ and $s$ for all larger angular disparities. Prediction error for each set of parameter values was evaluated as for Model II. The set of parameter values which maximized $P$ then comprised the estimates of $i, s, t$, and $k$.

Predicted group functions for each model were obtained by averaging the model's 20 predicted response times at each angle over the corresponding 20 subjects.

All computations were done on an Apple II + computer.

\section{Results and Discussion}

Each subject yielded parameter estimates and a value of $P$, the proportion of variance accounted for, for each model. The means of these measures are given in Table 1.

The ordering of the models by their goodness of fit matched an ordering of their complexity as indexed by number of parameters. This ordering, with Model III fitting best, followed by Model II and then Model I, was found for each subject. The relative quality of these fits is apparent in the group functions for the small-angle range, shown in Figure 2.

At the ordinal level, however, this result could have been foreseen. Because Model II is a special case of Model III (i.e., the case in which $k$ equals i), it must be possible for Model III to fit at least as well as Model II. Model III will fit better than Model II if the additional parameter $\mathrm{k}$ can assume a value that reduces residual variation, and this happens virtually all the time. Also, Model I is a special case of Model II (i.e., the case in which $t$ is zero), and a similar argument holds. For this reason, it would not be wise to accept the superior conventional fit of Model III by itself as empirical support for a new theory of mental rotation.

Table 1

Mean Parameter Estimates and Proportions of Variance Accounted For of Three Models Fit to Rossi (1980) Data

\begin{tabular}{rccccc}
\hline Model & i & $\mathrm{s}$ & $\mathrm{t}$ & $\mathrm{k}$ & $\mathrm{P}$ \\
\hline I & 1,034 & 14.4 & & & .775 \\
II & 1,118 & 13.7 & 11.9 & & .781 \\
III & 1,499 & 11.0 & 22.4 & 1,114 & .832 \\
\hline
\end{tabular}

Note $-i=$ intercept (in msec); $s=$ slope (in msec per degree); $t=$ threshold (in degrees); $k=$ subthreshold response time (in msec); $P=$ proportion of variance accounted for by the model.

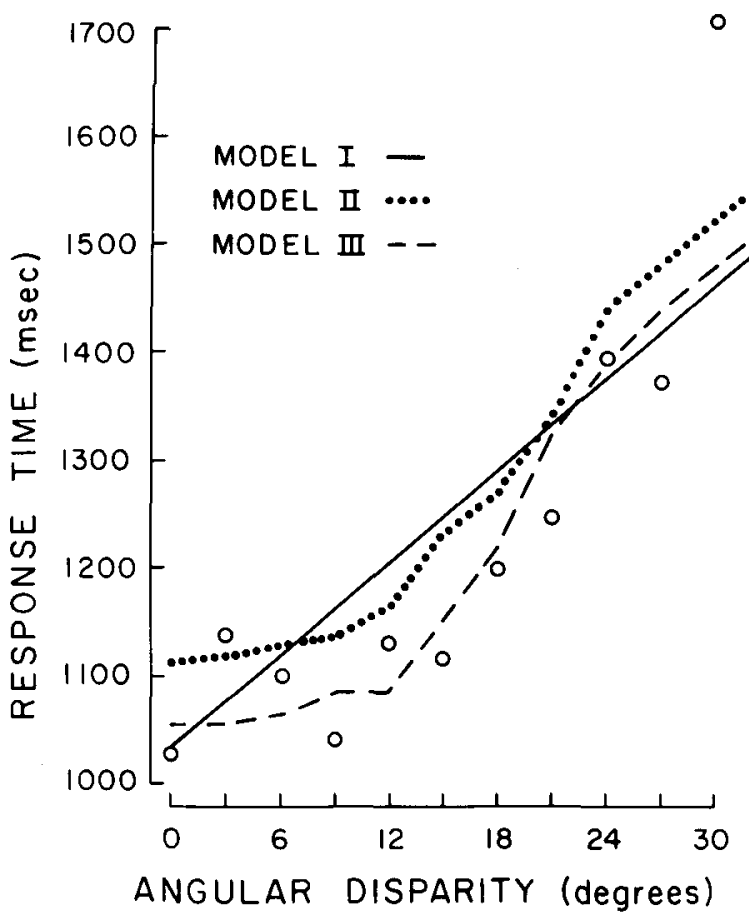

Figure 2. Group data from the original experiment (for the range of small angles only) and group predicted functions for each of the three models. Such predicted functions are obtained by averaging a fitted model's individual predicted functions over all subjects.

But neither is a dismissal of Model III and its possible theoretical interpretations forced upon us (although reverting to such a strength/fit option may seem especially attractive after this demonstration). There may actually be individual thresholds for mental rotation; the additional free parameters of Model III may measure an aspect of cognition, however imperfectly, as well as capitalize on the individual patterns of error. It seems worth emphasizing that the better fit of weaker models neither validates nor invalidates the underlying ideas.

The eventual acceptance or rejection of a model, in any case, should depend more on the reliability and usefulness of its parameter estimates than on its goodness of fit in a single experiment. Study 1 provides an example of how goodness of fit can be an empirically empty criterion for model comparisons. Before adopting an arbitrary strength/fit bias, drawing an uncertain conclusion, or going on to conduct further experiments with human subjects, we attempted to learn more about Models I, II, and III by simulating them in a computer program and then fitting them to the simulated data, a procedure termed "known-model analysis."

\section{STUDY 2 \\ Assessing the Sensitivity of Fitted Models Using Source-Model Controls}

The first step in this application of known-model analysis was to use the parameter estimates from a conven- 
tional analysis, such as the mean values in Table 1 , together with a source of error, to simulate many new sets of data. Each simulated set of data looks very much like the set of response times obtained from a human subject; however, the data set comes from a known source (the model programmed into the computer) and is calibrated by the original mean parameter estimates, so that, ostensibly, it represents typical performance by a subject who operates according to the known model. The second step was to fit each of the models to each of the simulated data sets. This achieved a full crossing (all models simulated by all models fit) of the two roles implicitly played by models in a conventional analysis: as candidate sources of the data and as theoretical structures fit to the data. The third step in the analysis was to extract information from the simulation-fit combinations about the sensitivity of fitted models to variations in the true source of the data. The ranking of the fitted models by their goodness of fit in our mental rotation example was known a priori to be insensitive to variation of the source model. How far this insensitivity would go in the actual pattern of model fits remained to be determined.

Models I, II, and III, as alternative sources of simulated data, can be thought of as "source-model controls." This term is meant to convey an analogy between simulations of known processes and the more familiar types of control conditions, such as no-treatment and placebo controls. A source-model control allows us to ask whether models fit as well when they are "wrong" as when they are "true."

Because the ability of models to capitalize on error to differing extents was a major concern in this analysis, the effect of error or noise level was also studied. Manipulation of the error component of the source models provided an opportunity to examine the sensitivity of fitted models as a function of the "signal-to-noise" (or, more precisely, "true-to-error") ratio in the simulated sets of data.

\section{Method}

An Apple II + microcomputer was programmed to simulate mental rotation response times under Models I, II, and III and to fit each of the models to each set of simulated data. The model simulations were based on the results of Study 1, in that "true" values of the parameters of each model were taken from, or were very close to, the mean estimates of these parameters in Table 1. The Model I simulation used $\mathrm{i}=1,034$ and $\mathrm{s}=14.4$. The Model II simulation used $\mathbf{i}=1,118, \mathrm{~s}=13.7$, and $\mathrm{t}=12$. The Model III simulation used $\mathrm{i}=1,499, \mathrm{~s}=11.0, \mathrm{t}=21$, and $\mathrm{k}=1,114$.

Each simulated response time was a single sample from a quasinormal distribution drawn by executing a version of the Box-Muller algorithm (Box \& Muller, 1958). In this algorithm, the computed values have the form

$$
Y=A+B\left[\sin \left(2 R_{1}\right)\right]\left(-2 \log _{e} R_{2}\right)^{3 / 2},
$$

where $\mathbf{R}_{\mathbf{1}}$ and $\mathbf{R}_{\mathbf{2}}$ are independent samples from a uniform distribution on the interval from 0 to 1 . Box and Muller showed that $Y$ is normally distributed with mean $A$ and standard deviation $B$. In the simulations, $R_{1}$ and $R_{2}$ were sampled from the Apple's randomnumber function for each simulated response time. (The RND function itself was randomly initialized by manual interrupt.) The standard deviation $B$ was set equal to 425 ; this value was close to the average standard error of estimate in linear-regression fits (Model I) to the individual data analyzed in Study 1 . The mean A was the response time predicted by the model being simulated, given the parameter values chosen for that model and a particular angular disparity.

One set of simulated data consisted of 16 response times corresponding to the 16 values of angular disparity used in the original mental rotation experiment. Five hundred such sets of data were generated under each of the three models. All three models were fit to each set of data, using the same procedures as in Study 1. The computer program kept track of several measures gleaned from each of the 4,500 combinations of a source model with a fitted model.

This known-model analysis was repeated with only one change in the design of the simulations: The standard deviation B was set equal to 42.5, one-tenth the standard error of estimate in linearregression fits to the original individual data sets. The analysis with $B=42.5$ will be termed the low-noise analysis; the one with $B=425$, the high-noise analysis.

\section{Results and Discussion}

The proportions (P) of variance accounted for by each of the three models in the known-model analyses and in the conventional analysis of Study 1 (in which the source was human) are shown in Figure 3.

The ranking of fitted models by goodness of fit was as foreseen rationally. For both the high-noise and low-noise analyses, Model III fit best, Model II next, and Model I last, regardless of the data's "true" source.

As the complexity of the source model increased, the fit of Models I and II declined, whereas the fit of Model III remained roughly the same, with $\mathrm{P}$ about .83 in the high-noise and about .998 in the low-noise analysis. Hence, Model III's goodness of fit was insensitive to differences among different sources of the data (including Model III, Model II, Model I, and, in the high-noise case, human subjects). It appears that although Model III's goodness of fit conveys gross information about the noise level in a set of data, it says nothing about the data's source.

The differences among fitted models, as the source model is varied, seem most informative in the low-noise analysis. A simple model's goodness of fit approached that of a more complex model, provided that the source model was also relatively simple. If the source model was more complex than the fitted model, goodness of fit declined. ${ }^{1}$ This pattern in the low-noise data is interesting because of its logical relation to one statistical approach to comparing simple and complex models. In multiple regression analysis, log-linear analysis, and some other statistical procedures, a simple model is discarded in favor of a more complicated one only if the resulting improvement in fit is statistically significant. Thus, if the more complicated model's fit is only marginally better, parsimony decides in favor of the simpler model. This statistical decision rule works well for the low-noise part of the known-model analysis, but not for the high-noise part. To see this, imagine that data symbols in Figure 3 that almost touch each other are not reliably different, whereas separated points differ significantly. The statistical decision rule applied to the low-noise data would iden- 


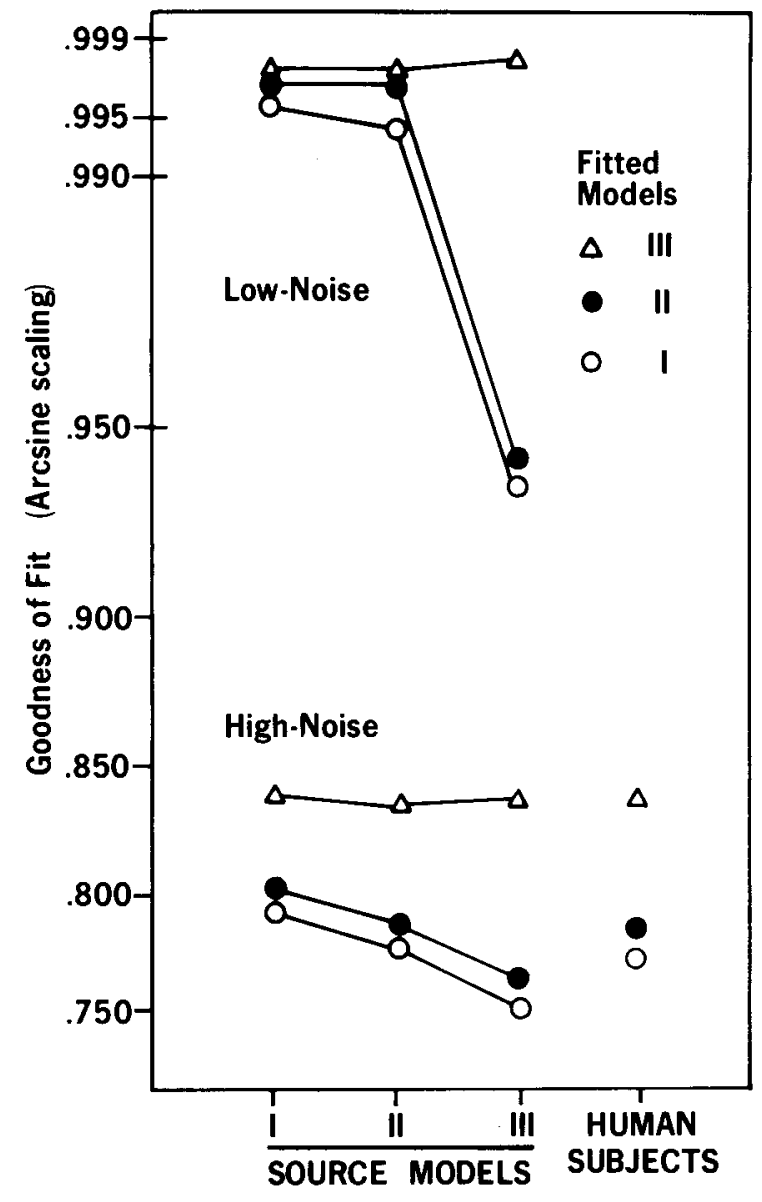

Figure 3. Mean goodness-of-fit values for each application of a fitted model to a source model at two levels of noise. Each such value is the mean of $\mathbf{5 0 0}$ proportions. Goodness-of-fit values from the conventional analysis (Study 1) of the human data are shown for comparison. Arcsine scaling on the vertical axis is used in order to show more clearly the pattern of fit values in the low-noise analysis.

tify each source model correctly. For example, Model III would be accepted only when the data source was Model III. However, in the high-noise analysis, Model III fits significantly better than its competitors, regardless of the data's source. This example illustrates how a high noise level can defeat a statistical decision rule; given noisy data and a model with a propensity to scavenge error variance, even an objective statistical rule will be insensitive to variations in the source of the data.

\section{GENERAL DISCUSSION}

Many psychologists recognize that if a model is made more and more complex, it will be able to explain more, although in a way that threatens the ideal of parsimony. At the same time, the working assumption that "the true model will fit best" is widespread among scientists in many disciplines. This paper is located at a point where these cognitions cease to coexist peacefully and begin to clash with one another. Weak models generally fit better than strong models, and this undermines the use of fit as a guide to choosing a source model.

The original mental rotation data collected by Rossi came from an "unknown" (but generically human) source. Figure 3 shows that this source yielded its data in a noise-level range in which fitted models cannot discriminate among source models, even if a statistical test is applied to the improvements in fit achieved by increasing fitted-model complexity. This finding, unlike the rational argument concerning the ordering of models, is an empirical one. Known-model analysis can, in principle, be used to investigate the sensitivity of fitted models to source-model variations, not only for the "nested" families of models represented here by Models I, II, and III, but for any set of well-defined models.

The use of known-model analysis to investigate the sensitivity of fitted models can be likened to the use of a control group to provide a point of comparison in assessing treatments. Researchers understand that positive results from a treatment do not alone establish the effectiveness of the treatment; in order to claim that an effect has been found, the researcher must show that these results do not also occur without treatment or with a different treatment. Similarly, the finding that one model fits a set of data better than competing models does not establish the model as the probable source of the data. To support such a claim, the researcher must show that the model does not also fit better when, as a source model, it is "false." Known-model analysis can provide this source-model control information. In fact, source-model controls are practical only because computer simulations of known models are possible, and they represent an important way in which computers can extend the application of scientific method.

Some limitations of the present analysis must be noted. Each of the three models was simulated by adding Gaussian noise to only one exemplar of the model. Although these exemplars were chosen on the basis of averaged parameter estimates derived from human data, the singleexemplar approach is still a short-cut. It substitutes the Gaussian noise for explicit models of both within-subject error and individual differences. It also assumes that independently averaged parameter estimates can describe a representative individual for the purposes of model comparison, and this assumption may be inappropriate. These problems will have to be addressed in further research with models of mental-rotation performance.

The superior fit of Model III to individual subjects' data has no real substantive implications, as we have seen. However, this does not mean that the hypothesis of a threshold for mental rotation is impossible to test. Rossi and Collyer (in press) have suggested that a thresholdlike response-time function may be the result of presenting the small angles of stimulus rotation more frequently than the larger angles, a possible "range-frequency effect" (see Parducci, 1974) in response-time data. A threshold, whether or not it is induced by a nonuniform distribution of stimulus angles, may be apparent in averaged group data but not in the relatively noisier data of 
individual subjects. Paradoxically, some concepts that make rational sense only at the level of the individual, such as thresholds for mental rotation, may be empirically and practically demonstrable only in group data.

One lesson we have learned from this research is that we must be cautious in the interpretation of goodness of fit measures. One reason for caution is the need for source-model control information to determine whether goodness of fit can be taken as an indicator of the data's source. This need seems particularly important in the comparison of strong and weak models. Another reason for caution is the possible futility of using goodness of fit to compare precise theoretical models at the level of individual subjects, where the noise levels may both obliterate testable differences among the models of interest and guarantee significantly better fit for the weakest model. Known-model analysis provides one means of addressing both model sensitivity and testability.

Known-model analysis and a number of related procedures such as cross-validation (Efron \& Gong, 1983; Mosier, 1951) and bootstrapping (Diaconis \& Efron, 1983) represent relatively new ways of using computers to help solve scientific problems. To date, most of the work on these procedures has been done in the fields of statistics and psychometrics. Our laboratory is currently exploring these procedures and some of their applications to topics in general experimental psychology.

\section{REFERENCES}

Box, G. E. P., \& Muller, M. E. (1958). A note on the generation of random normal variates. Annals of Mathematical Statistics, 29, 610-611.

Bush, R. R., \& Mosteller, F. (1955). Stochastic models for learning. New York: Wiley.
COOPER, L. A., \& SHEPARD, R. N. (1978). Transformations on representations of objects in space. In E. C. Carterette \& M. P. Friedman (Eds.), Handbook of perception (Vol. 8). New York: Academic Press. Diaconis, P., \& EFron, B. (1983). Computer-intensive methods in statistics. Scientific American, 249 (May).

EFroN, B., \& GoNG, G. (1983). A leisurely look at the bootstrap, the jackknife, and cross-validation. The American Statistician, 37, 36-48.

Mosier, C. I. (1951). Problems and designs of cross-validation. Educational \& Psychological Measurement. 11, 5-11.

PARDUCCI, A. (1974). Contextual effects: A range-frequency analysis. In E. C. Carterette \& M. P. Friedman (Eds.), Handbook of perception (Vol. 2). New York: Academic Press.

Pedhazur, E. J. (1983). Multiple regression in behavioral research (2nd ed.). New York: Holt, Rinehart \& Winston.

Pylyshyn, Z. W. (1979). The rate of "mental rotation" of images: A test of a holistic analogue hypothesis. Memory \& Cognition, 7, 19-28.

Rossi, J. S. (1980). Is there a threshold for mental rotation? Master's thesis, University of Rhode Island.

Rossi, J. S., \& Collyer, C. E. (in press). Is there a threshold for mental rotation? Bulletin of the Psychonomic Society.

ShePARD, R. N., \& METzleR, J. (1971). Mental rotation of threedimensional objects. Science, 171, 701-703.

WagenaAR, W. A., \& Padmos, P. (1971). Quantitative interpretation of stress in Kruskal's multidimensional scaling technique. British Journal of Mathematical \& Statistical Psychology, 24, 101-110.

\section{NOTE}

1. The pattern of fits in the low-noise data shown in Figure 3 is analogous to the "elbow effect" in multidimensional scaling (MDS) Wagenaar and Padmos (1971) discussed a computer simulation study on the estimation of dimensionality and measurement error in MDS which illustrates the elbow effect within the context of a known-model design similar to the present one. They note that the effect is pronounced only for low-stress (low-noise) data.

(Manuscript received December 13, 1984; revision accepted for publication October 31,1985 .) 